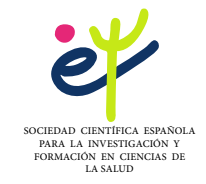

\title{
Conexiones y desconexiones morales frente al bullying entre alumnado con y sin Sistemas de Apoyo entre Iguales
}

\section{Moral connections and disconnections to bullying among students with and without Peer Support Systems}

\author{
José Maria Avilés Martínez ${ }^{1^{*}}$ (D) \& Luciene Regina Paulino Tognetta² \\ ${ }^{1}$ Universidad de Valladolid (España), ${ }^{2}$ Universidade Estadual Paulista (Brasil)
}

\section{RESUMEN}

Introducción: Los escenarios de maltrato entre iguales obligan a adoptar posturas morales a quienes participan en ellos respecto de lo que sucede y a sus actores. El discurso educativo de la escuela ayuda a conformar posiciones de inhibición o rechazo del abuso. Además, el protagonismo del alumnado a través de Sistemas de Apoyo entre Iguales se manifiesta como una estrategia de prevención eficaz para su prevención.

Método: Esta investigación compara una muestra de sujetos españoles y brasileños, escolarizados en centros con y sin Sistemas de Apoyo entre Iguales a quienes se pide su posicionamiento moral ante dos situaciones de bullying que muestran víctimas de distinto perfil, pasivo y provocador. Sus respuestas se clasifican en niveles de calidad moral diferente.

Resultados: El alumnado que dispone de estructuras de apoyo entre iguales ofrece mejores posicionamientos morales. También se dan mejores respuestas entre el alumnado español que en el brasileño.

Conclusión: Fomentar estructuras de apoyo entre iguales entre el alumnado facilita su crecimiento moral ante situaciones de bullying y es una estrategia preventiva eficaz para mejorar la convivencia en los centros escolares.

Palabras Clave: Sistemas de Apoyo entre Iguales, Bullying, Convivencia, Equipos de Ayuda, Victimización, Educación Moral.

\footnotetext{
* Autor de Correspondencia: Dr. José María Avilés Martínez, (aviles@uva.es). Facultad de Educación y Trabajo Social, Universidad de Valladolid. Calle Paseo de Belén 1, Campus Miguel Delibes 47011 Valladolid (España).
} 


\begin{abstract}
Introduction: Situations of peer bullying force to adopt moral positions among those who participate in them. The educational message of the school helps to form positions of inhibition or rejection of abuse. In addition, the leadership (presence)? of students through Support Systems is manifested as an effective prevention strategy for its prevention.
\end{abstract}

Method: This research compares a sample of Spanish and Brazilian subjects, in schools with and without support systems who are asked for their moral positioning in front of two situations of bullying that show victims of different profiles, passive and provocative. Your answers are classified in different levels of moral quality.

Results: The students who have peers support structures offer better moral positions. There are also better responses among Spanish students than in Brazilian ones.

Conclusion: Promote peer support structures whit the students facilitate their moral growth in situations of bullying and is an effective preventive strategy to improve convivencia in schools.

Keywords: Peer support, Bullying, Convivencia, Help Team, Victimizationa, Moral Education.

\title{
INTRODUCCIÓN
}

\section{El bullying como problema moral}

Conocida es la vertiente moral que descansa en las dinámicas bullying y que ha detraído investigaciones en la literatura científica (Avilés, Tognetta y Petta, 2020; Gini, 2006, 2020; Tognetta, Avilés y Rosario, 2016). Especialmente relevantes para las personas implicadas en ellas han sido los posicionamientos que han adoptado según su perfil de participación. Mientras los agresores utilizan mecanismos justificativos, desplazadores, minimizadores y/o culpabilizadores de sus víctimas en las formas exculpatorias que adoptan para autoexonerarse, quienes contemplan los hechos también toman partido por unos u otros o se muestran indiferentes, lo que indica lo que piensan de los implicados y de la propia dinámica del maltrato entre iguales. Incluso, las víctimas se explican lo que sucede y sus actuaciones echando mano de argumentos de rechazo y/o evitación, o en el peor de los casos, especialmente entre las de larga duración, también de autoculpabilización, al no encontrar una explicación razonable de las posturas de sus compañeros que les agreden o que se inhiben. Por acción o por omisión, la dinámica del maltrato entre iguales sitúa a cada perfil en una posición social dentro del grupo, pero también en una opción moral ante lo que pasa.

Bandura $(2002,2016)$ explica diversos mecanismos psicológicos de descarga moral para hacer soportables situaciones que desde el punto de vista objetivo no lo son, y esto también en el caso del bullying, explica por qué personas buenas son capaces de hacer cosas 
malas que perjudican a otras o a ellas mismas, por actuación, descuido o indiferencia. Son las llamadas desconexiones morales. Podríamos pensar que son mecanismos para situarse ante el bullying (autoprotección, exoneración, inhibición, derivación, ...), sin que la propia dinámica consiga despertar en el individuo conciencia negativa de sus acciones.

Estos mecanismos no son nuevos y se construyen en las trayectorias de historia personal ligadas a formas socializadoras de trivialización y consolidación social del fenómeno, apoyadas en figuras vinculares para el menor como son su propia familia o sus amistades. Este aprendizaje ha sido a su vez vinculado a emociones morales como la vergüenza o la culpa, tan presentes en la dinámica bullying en víctimas o en testigos.

Esto nos lleva a analizar esta amalgama de emociones en un escenario social de fuerzas, de forma que si el valor que predomina en el grupo es el de dominio-sumisión, compiten por prevalecer cuando el sometimiento del débil es el discurso vigente en la convivencia del grupo. Por esto, si en el grupo no se fomenta otra suerte de valores, los sujetos que tienen poder suelen implantar su discurso imponiendo una línea, a la que sus miembros suelen adaptarse en un ejercicio de ajuste por presión, si no hay ninguna voz discordante o contraria, o si en la institución no se promocionan discursos diferentes. Así se dibujan los roles y las dependencias en el seno del grupo.

Sin embargo, las personas adultas que conviven con el grupo siempre tienen la oportunidad de brindar estructuras estables que sirvan de tramas reconocidas y visibles que promocionen entre el alumnado el discurso del apoyo, la ayuda y otros valores que la propia institución tenga en su proyecto educativo de convivencia. Eso puede suponer un salto cualitativo de actuación para las escuelas y su alumnado, que encuentran una vía institucional para canalizar mensajes y acciones de mejora de la convivencia. Cualitativamente importante y diferente es el cambio que puede suponer en el plano individual para quienes se implican ayudando a otros y para quienes son ayudados en dinámicas de acoso. A unos y otros les supone cambiar la perspectiva que hasta ahora habían tenido en esas dinámicas y fomentar actuaciones nuevas, donde se plantean salidas alternativas, con mensajes de apoyo y ayuda a quienes lo necesitan dentro del grupo. Quienes agreden pasan de ser considerados poderosos a ser sujetos reprobables socialmente. Quienes son agredidos pasan de ser sujetos culpables de lo que les pasa, a ser considerados sujetos de ayuda o personas que tienen necesidades que solventar. Por último, el mensaje de la escuela activa la pasividad de quienes miran comprometiéndolos en no quedarse impasibles ante lo que ven. Cambiar esas inercias, imágenes de sí y posiciones morales en el seno del grupo, activa la autodefensa y la defensa colectiva, haciendo presente un discurso predominante, preventivo y protector del abuso.

\section{Las lógicas de acción de la dinámica bullying}

Es necesario que todas las personas sean conscientes del lugar que ocupan en el escenario del acoso, de la imagen que proyectan de sí y de otras personas y reflexionen sobre la lógica 
del abuso cuando sucede. Sin embargo, las dinámicas de acoso no se producen de forma única, cada caso es particular, ni se viven igual desde todas las perspectivas.

Especialmente en el agresor funciona una lógica de acción que permite elegir a un igual como medio para obtener sus objetivos sociales o, incluso, para satisfacer sus deseos de superioridad, haciendo gala de valores especialmente individualistas frente a los valores morales, como manifestación de su propia personalidad moral (Tognetta y Rosário, 2013). Por eso, no causa sorpresa la afirmación de Sutton, Smith y Swettenham (1999) cuando observan en los agresores respuestas argumentativas ante sus actos, de sentirse así poderosos o de parecer mejores que sus compañeros. Alentadas también por quienes les acompañan y que a su vez se han posicionado moralmente ante los hechos, cuando sonríen o incitan verbalmente sus acciones directas (Salmivalli y Voeten, 2004), o cuando transmiten actitudes que demuestran aprobación de sus conductas. Algo indicativo del refuerzo social positivo de las conductas intimidatorias (Juvonen y Galvan, 2008).

También quienes se acostumbran a mirar hacia otro lado sin hacer nada se posicionan moralmente ante los hechos al no emitir la desaprobación de lo que pasa, porque en definitiva transmiten a quienes agreden una indiferencia que sirve de aval a sus acciones agresoras (Del Barrio, Gutiérrez, Barrios, van der Meulen y Granizo, 2005; Salmivalli y Peets, 2009).

Pero es que el posicionamiento moral afecta incluso a la propia víctima. Acumula explicaciones insatisfactorias de lo que le sucede a partir de la imagen que ella y los demás tienen de si misma en esos escenarios, y que aumenta su sufrimiento y sus emociones morales de vergüenza y culpa. Los hechos continuados la sitúan como causa de lo que pasa y le provocan desconexiones morales de culpabilización (Avilés, 2019b; Tognetta, Avilés y Rosario, 2016) que le impiden superarlo. De ahí, la importancia de las emociones morales frente al bullying (Gini, 2006; Gini, Pozzoli y Hauser, 2011).

Por tanto, podemos establecer una interdependencia entre los procesos cognitivos, emocionales y sociales implicados en el acoso y las valoraciones morales que los sujetos hacen. Mecanismos que configuran los propios casos y condicionan las relaciones interpersonales y las atribuciones sociales a esas formas (Díaz-Aguado, 2006). De ahí la importancia de trabajar con el alumnado el plano moral para hacerles conscientes de sus posiciones, despertando compromisos a partir de valores ayuda, respeto y de rechazo del abuso y la dominación.

\section{El andamiaje institucional y su contribución a la educación moral frente al bullying}

Hemos de reconocer la importancia que la escuela tiene como difusora de un discurso preventivo del bullying para una contrucción de relaciones interpersonales entre iguales sanas y saludables. Por eso, el discurso institucional de la escuela es básico a distintos niveles. 
Como comunidad educativa y centro, el mensaje que trasmita la escuela debe consagrar la convivencia respetuosa como base para el aprendizaje y como eje del bienestar personal y social. Su ethos tiene que promocionar valores como el respeto, la ayuda, la solidaridad y el valor de las diferencias individuales como base de la convivencia.

En el grupo aula tiene que consolidarse un trabajo intencional y curricular donde los valores morales deben ser educados en colaboración con las familias. El escenario social del aula debe ser el espacio de práctica donde se pongan en juego y la convivencia familiar no debe ser sino su confirmación de respaldo. La escuela debe poner en debate y cuestión las decisiones individuales y colectivas que sobre el acoso surgen, cómo enfrentar la presión del grupo cuando hay fuerzas contrapuestas que luchan por dominar, cómo construir normas antibullying que ayuden a quienes son victimizados o cómo hacer prevalecer las decisiones moralmente más justas conforme a un código de valores establecido. Sin embargo, colectivamente, el aula debe dotarse de redes de apoyo que permitan recurrir a ellas cuando los problemas surjan y se hagan difíciles de resolver. Así, el profesorado es responsable de facilitar esas estructuras de forma consciente e intencional. Cada grupo de convivencia debe disponer de una red de iguales, reconocida y respaldada por el propio grupo que asista a cualquiera de sus miembros en momentos de difícultad. Esto se ha mostrado exitoso para mejorar la convivencia y el clima de aula. Las modalidades que adopta y la versatilidad que ofrece son importantes y cada centro puede adaptarlas a sus propias necesidades. Es lo que se ha denominado Sistemas de Apoyo entre Iguales -SAIs- (Avilés, 2017).

Estas estructuras desde dentro del grupo permiten construir y educar el posiconamiento moral de los sujetos ante el bullying a través de los iguales en positivo. Permiten trasmitir el discurso de la escuela en la práctica relacional del aula. Prestar el servicio de la ayuda de igual a igual como discurso dominante en las interacciones. Supone llenar los silencios cómplices del acoso con palabras contrarias a él que tienen valor positivo de cohesión de grupo.

\section{Una propuesta organizativa que soporte y difunda el discurso}

Los Equipos de Ayuda (Avilés, Torres y Vián, 2008) son uno de esos SAIs que tienen el valor de la ayuda como eje fundamental de su tarea. Se trata de equipos de tres o cuatro alumnos y alumnas que son elegidos por sus iguales para prestar ese servicio en cada una de las aulas. Previamente a hacer la ayuda se les prepara haciendo que reciban una formación que les proporciona el profesorado de su propio centro, previamente capacitado, a partir de un curriculo definido que incluye funciones y compromisos que adquieren, estrategias específicas para prestar la ayuda, conocimiento de los conflictos, el acoso y su dinámica, formas de comunicación, escucha activa, asertividad y empatía y un módulo específico de trabajo y toma de decisiones en equipo. De esta forma se constituyen en estructuras colectivas que son supervisadas por las personas adultas del centro que trabajan la convivencia. En 
equipo toman decisiones de convivencia y prestan apoyos a iguales que presentan alguna necesidad, sea de la naturaleza que sea, excepto en situaciones de drogas, abuso sexual o riesgo para la vida, casos en que saben que tienen que romper su confidencialidad para derivarlos a alguna persona adulta que los maneje.

Los Equipos de Ayuda son, por tanto, un soporte organizativo que permite el empoderamiento responsable del alumnado para canalizar de manera cercana y práctica los valores y contenidos éticos que están detrás de un plantemaiento educativo de convivencia en positivo. De forma proactiva, reducen el escalamiento de los conflictos y se constituyen como una barrera preventiva de gran eficacia para hacer presente la conciencia colectiva sobre el acoso, su impacto emocional en las víctimas y la gestión eficaz de situaciones de bullying en el seno de los grupos (Avilés y Petta, 2018)

\section{OBJETIVOS}

La investigación plantea la hipótesis de que el alumnado formado en contextos educativos donde se estimulan de forma intencional los valores de la ayuda a través de Equipos de Ayuda tendrá un desarrollo moral superior ante el bullying que otro que no disponga de esas estructuras explícitamente.

Se valoran las respuestas del alumnado ante dos situaciones de bullying que se les presentan, comparando estudiantes brasileños y españoles que en sus centros tienen la estructura de Equipos de Ayuda (CONEA) y otros que no disponen de ese sistema (SINEA).

\section{MÉTODO}

\section{Participantes}

Son 1068 alumnos y alumnas de centros educativos de Brasil (630) y España (438). El alumnado español pertenece a centros públicos de A Coruña, Barcelona, Guadalajara, Madrid y Valladolid. El brasileño es de centros públicos y privados del estado de Sao Paulo.

La muestra se distribuye en dos grupos, tanto en Brasil como en España. El primero es alumnado que en sus centros dispone de estructuras de Equipos de Ayuda, en adelante CONEA. El segundo no dispone en sus centros de Equipos de Ayuda, en adelante SINEA, conforme se muestra en la Tabla 1. La distribución por sexos es similar en ambos países.

La diferencia muestral CONEA y SINEA es que solo los primeros reciben formación específica en convivencia para desempeñar su función de Equipos de Ayuda durante dos días mediante un paquete formativo compartido (Avilés y Alonso, 2017) en los dos países y escuelas. 
Tabla1. Distribución por países y sexos.

\begin{tabular}{|c|c|c|c|c|c}
\hline & \multicolumn{2}{|c|}{ España } & \multicolumn{2}{c|}{ Brasil } & \\
\hline & Chicos & Chicas & Chicos & Chicas & Total \\
\hline CONEA & $123(51.4 \%)$ & $120(48.6 \%)$ & $259(51.8 \%)$ & $241(48.2 \%)$ & $743(69.57)$ \\
\hline SINEA & $107(54.9 \%)$ & $88(45.1 \%)$ & $74(56.92 \%)$ & $56(43.08 \%)$ & $325(30.43)$ \\
\hline Total & $230(52.52 \%)$ & $208(47.48 \%)$ & $333(52.86 \%)$ & $297(47.14 \%)$ & $1068(100 \%)$ \\
\hline
\end{tabular}

El alumnado que participa en las dos categorías aparece reflejado en la Tabla 2.

Tabla 2: Distribución del alumnado por países y centros con y sin Equipos de Ayuda.

\begin{tabular}{|c|c|c|c|}
\hline & Brasil & España & Total \\
\hline SINEA & $130(20.7 \%)$ & $195(44.6 \%)$ & $325(30.43 \%)$ \\
\hline CONEA & $500(79.3 \%)$ & $243(55.4 \%)$ & $743(69.57 \%)$ \\
\hline Total & $630(100 \%)$ & $438(100 \%)$ & 1068 \\
\hline
\end{tabular}

\section{Instrumentos y procedimiento}

$\mathrm{Al}$ alumnado se le enfrenta a un instrumento para recoger sus posiciones morales ante el bullying validado en otras investigaciones (Tognetta y Rosario, 2013; Tognetta, Avilés, Rosario y Alonso, 2015) que obtiene sus respuestas ante dos relatos de bullying, uno de víctima pasiva (HA) y otro de provocativa (HB). Se ofrecen ocho alternativas en cada historia, eligiendo aquellas con las que están de acuerdo. Cada alternativa responde a opciones de conexión y desconexión moral conforme a los postulados de la epistemología genética (Kohlberg, 1992; Piaget, 1932) y a la categorización señalada por Bandura (1999).

\section{Diseño y Análisis Estadístico}

Las respuestas se categorizan resultando 4 factores (subdivididos en F1, F2, F3 e F4), que resumen las opciones de conexión y desconexión. Cada puntaje (factor) se define por el porcentaje de alternativas señaladas entre las preguntas de cada subgrupo.

A partir de las puntuaciones obtenidas se formaron grupos de sujetos en función de las respuestas positivas en cada factor. Así, el grupo F1, F2, F3 y F4 estuvo formado por 
personas que tuvieron respuestas positivas en los cuatro factores, mientras que el grupo F1 estaba formado por personas que solo ofrecieron respuestas positivas en ese factor. Las combinaciones resultantes se reflejan en la Tabla 3 por países.

Tabla 3: Agrupación de las respuestas de los sujetos en cada factor.

\begin{tabular}{|c|c|c|c|}
\hline BRASIL & ESPAÑA & BRASIL & ESPAÑA \\
\hline \multicolumn{2}{|c|}{ Grupo (HA) } & \multicolumn{2}{|c|}{ Grupo (HB) } \\
\hline F1 & F1 & F1 & F1 \\
\hline $\mathrm{F} 1, \mathrm{~F} 2$ & F1, F2 & F1, F2 & $\mathrm{F} 1, \mathrm{~F} 2$ \\
\hline $\mathrm{F} 1, \mathrm{~F} 2, \mathrm{~F} 3$ & F1, F2, F3 & F1, F2, F3, F4 & F1, F2, F3 \\
\hline $\mathrm{F} 1, \mathrm{~F} 2, \mathrm{~F} 3, \mathrm{~F} 4$ & $\mathrm{~F} 1, \mathrm{~F} 2, \mathrm{~F} 3, \mathrm{~F} 4$ & $\mathrm{~F} 1, \mathrm{~F} 2, \mathrm{~F} 4$ & F1, F2, F3, F4 \\
\hline $\mathrm{F} 1, \mathrm{~F} 2, \mathrm{~F} 4$ & $\mathrm{~F} 1, \mathrm{~F} 2, \mathrm{~F} 4$ & F1, F3 & $\mathrm{F} 1, \mathrm{~F} 2, \mathrm{~F} 4$ \\
\hline F1, F3 & F1, F3 & F1, F3, F4 & F1, F3 \\
\hline $\mathrm{F} 1, \mathrm{~F} 3, \mathrm{~F} 4$ & F1, F3, F4 & F1, F4 & $\mathrm{F} 1, \mathrm{~F} 3, \mathrm{~F} 4$ \\
\hline $\mathrm{F} 1, \mathrm{~F} 4$ & $\mathrm{~F} 1, \mathrm{~F} 4$ & $\mathrm{~F} 2$ & $\mathrm{~F} 1, \mathrm{~F} 4$ \\
\hline $\mathrm{F} 2$ & $\mathrm{~F} 2$ & $\mathrm{~F} 2, \mathrm{~F} 3$ & $\mathrm{~F} 2$ \\
\hline $\mathrm{F} 2, \mathrm{~F} 3$ & $\mathrm{~F} 2, \mathrm{~F} 3$ & $\mathrm{~F} 2, \mathrm{~F} 3, \mathrm{~F} 4$ & F2, F3 \\
\hline $\mathrm{F} 2, \mathrm{~F} 3, \mathrm{~F} 4$ & $\mathrm{~F} 2, \mathrm{~F} 3, \mathrm{~F} 4$ & $\mathrm{~F} 2, \mathrm{~F} 4$ & $\mathrm{~F} 2, \mathrm{~F} 3, \mathrm{~F} 4$ \\
\hline $\mathrm{F} 2, \mathrm{~F} 4$ & $\mathrm{~F} 2, \mathrm{~F} 4$ & F3 & $\mathrm{F} 2, \mathrm{~F} 4$ \\
\hline F3 & F3 & $\mathrm{F} 3, \mathrm{~F} 4$ & F3 \\
\hline F3, F4 & $\mathrm{F} 3, \mathrm{~F} 4$ & F4 & $\mathrm{F} 3, \mathrm{~F} 4$ \\
\hline F4 & F4 & & F4 \\
\hline
\end{tabular}

Tras la comprobación estadística de viabilidad de los factores establecidos se llega a cuatro niveles progresivos de desarrollo moral conforme a las respuestas emitidas. Estos niveles se forman a partir de los agrupamientos de respuestas a esos factores de los grupos que aparecen en la Tabla 3. Por ejemplo, el Nivel 1 contiene personas que solo eligieron respuestas de F1, F2 o en ambos conforme a la distribución que aparece en la Tabla 4.

Tabla 4: Niveles para cada historia (HA y HB) a partir de sus factores agrupados.

Nivel 1 - F1; F2; F1 y F2

Nivel 2 - F1 y F3; F1 y F4; F2 y F3; F1, F2 y F3; F1, F2 y F4; F1, F3 y F4; F1, F2, F3 y F4

Nivel 3 - F2 y F4; F2, F3 y F4

Nivel 4 - F3; F4; F3 y F4 
- Nivel 1 correspondiente a categorías de desconexión moral que niegan el propio contenido moral de la situación, culpabilizando o deshumanizando a la víctima o justificando o disfrazando lo que sucede.

- Nivel 2 representa respuestas que revelan desconexión moral, aunque sin la negación del contenido moral en juego, desplazando o difundiendo responsabilidad, o minimizando o distorsionando consecuencias para la víctima.

- Nivel 3 engloba respuestas que poseen conexión moral, aunque por convención social.

- Nivel 4 identifica las respuestas propiamente morales por principio.

El análisis estadístico se hizo con software SAS 9.2. adoptándose en las asociaciones un nivel de significación del 5\%. Las asociaciones entre niveles y grupo (CONEA y SINEA) y entre preguntas y grupo, así como entre niveles y país se analizaron a través de Chi cuadrado.

\section{RESULTADOS}

Al comparar globalmente respuestas morales del alumnado ante el bullying hay más alumnado conectado (Niveles 3 y 4) que desconectado (Niveles 1 y 2) moralmente ante el bullying en centros CONEA que en centros SINEA, tanto en Brasil como en Espańa. Igualmente, se encuentran diferencias significativas entre centros CONEA y SINEA, aunque estas diferencias se constatan significativas en Brasil, pero no en España.

Como muestra la Tabla 5, al comparar los centros brasileńos CONEA y SINEA los niveles de desarrollo moral más elevados (Nivel 3 y 4 ) se dan en los primeros, tanto ante víctima pasiva como provocativa, y los más bajos (Nivel 1 y 2), en los últimos.

Tabla 5: Posturas morales del alumnado ante el bullying en centros brasileños con y sin Equipos de Ayuda.

\begin{tabular}{|c|c|c|c|c|}
\hline \multicolumn{5}{|c|}{ BRASIL } \\
\hline Grupo $(H A)$ & CONEA & SINEA & TOTAL & valor- $p$ \\
\hline Nivel 1 & $17(3.69 \%)$ & $8(6.15 \%)$ & $25(4.23 \%)$ & \multirow{4}{*}{$<0.01$} \\
\hline Nivel 2 & $96(20.82 \%)$ & $71(54.62 \%)$ & $167(28.26 \%)$ & \\
\hline Nivel 3 & $140(30.37 \%)$ & $22(16.92 \%)$ & $162(27.41 \%)$ & \\
\hline Nivel 4 & $208(45.12 \%)$ & $29(22.31 \%)$ & $237(40.1 \%)$ & \\
\hline \multicolumn{5}{|l|}{ Grupo (HB) } \\
\hline Nivel 1 & $26(5.62 \%)$ & $12(9.23 \%)$ & $38(6.41 \%)$ & \multirow{4}{*}{$<0.01$} \\
\hline Nivel 2 & $158(34.13 \%)$ & $80(61.54 \%)$ & $238(40.13 \%)$ & \\
\hline Nivel 3 & $77(16.63 \%)$ & $13(10 \%)$ & $90(15.18 \%)$ & \\
\hline Nivel 4 & $202(43.63 \%)$ & $25(19.23 \%)$ & $227(38.28 \%)$ & \\
\hline
\end{tabular}


En cambio, como se observa en la Tabla 6 los niveles de calidad de las posturas morales ante el bullying tomados globalmente, tanto en situaciones con víctimas pasivas como con víctimas provocativas, no ofrecen diferencias significativas entre el alumnado español, sea en centros CONEA como en centros SINEA.

Tabla 6: Posturas morales del alumnado ante el bullying en centros españoles con y sin Equipos de Ayuda.

\begin{tabular}{|c|c|c|c|c|}
\hline \multicolumn{5}{|c|}{ ESPAÑA } \\
\hline Grupo (HA) & CONEA & SINEA & TOTAL & \\
\hline Nivel 1 & $17(3.7 \%)$ & $7(6.8 \%)$ & $24(4.27 \%)$ & \multirow{4}{*}{$<0.31$} \\
\hline Nivel 2 & $170(37.04 \%)$ & $33(32.04 \%)$ & $203(36.12 \%)$ & \\
\hline Nivel 3 & $123(26.8 \%)$ & $33(32.04 \%)$ & $156(27.76 \%)$ & \\
\hline Nivel 4 & $149(32.46 \%)$ & $30(29.13 \%)$ & $179(31.85 \%)$ & \\
\hline \multicolumn{5}{|l|}{ Grupo (HB) } \\
\hline Nivel 1 & $11(2.4 \%)$ & $6(5.83 \%)$ & $17(3.02 \%)$ & \multirow{4}{*}{$<0.21$} \\
\hline Nivel 2 & $200(43.57 \%)$ & $49(47.57 \%)$ & $249(44.31 \%)$ & \\
\hline Nivel 3 & $97(21.13 \%)$ & $20(19.42 \%)$ & $117(20.82 \%)$ & \\
\hline Nivel 4 & $151(32.9 \%)$ & $28(27.18 \%)$ & $179(31.85 \%)$ & \\
\hline
\end{tabular}

Por otra parte, si comparamos los resultados por países encontramos diferencias significativas en los niveles de desarrollo moral, tanto si consideramos aisladamente el alumnado de centros SINEA como de centros CONEA. Esas diferencias se dan tanto ante situaciones en que interviene una víctima pasiva como en las que lo hace una de perfil provocador.

En los centros CONEA, es en España, como aparece en la Tabla 7, donde se agrupa más alumnado en los niveles más avanzados de desarrollo moral (3 y 4), ya sea juzgando el bullying con víctima pasiva como provocativa. El perfil provocador en la víctima se entiende moralmente algo mejor en España que en Brasil, donde según la Tabla 7 se acumula más de la mitad de la muestra en el nivel 2 de desconexión moral, a pesar de tratarse de centros donde existen Equipos de Ayuda. No obstante, este tipo de víctima en relación con la pasiva despierta menor comprensión en ambos países, aunque haya estructuras de ayuda entre su alumnado. 
AVILÉS \& TOGNETTA. Conexiones y desconexiones morales frente al bullying entre alumnado...

Tabla 7: Posturas morales ante el bullying entre países en centros con estructuras de Equipos de Ayuda.

\begin{tabular}{|c|c|c|c|c|}
\hline \multirow{2}{*}{ Historia } & \multicolumn{3}{|c|}{ CONEA } & \multirow{2}{*}{ Valor-p* } \\
\hline & Brasil & España & Total & \\
\hline \multicolumn{5}{|c|}{ Grupo (HA) } \\
\hline Nivel 1 & $8(6.15 \%)$ & $7(6.8 \%)$ & $15(6.44 \%)$ & \multirow{4}{*}{$<0.01$} \\
\hline Nivel 2 & $71(54.62 \%)$ & $33(32.04 \%)$ & $104(44.64 \%)$ & \\
\hline Nivel 3 & $22(16.92 \%)$ & $33(32.04 \%)$ & $55(23.61 \%)$ & \\
\hline Nivel 4 & $29(22.31 \%)$ & $30(29.13 \%)$ & $59(25.32 \%)$ & \\
\hline \multicolumn{5}{|c|}{ Grupo (HB) } \\
\hline Nivel 1 & $12(9.23 \%)$ & $6(5.83 \%)$ & $18(7.73 \%)$ & \multirow{4}{*}{0.04} \\
\hline Nivel 2 & $80(61.54 \%)$ & $49(47.57 \%)$ & $129(55.36 \%)$ & \\
\hline Nivel 3 & $13(10 \%)$ & $20(19.42 \%)$ & $33(14.16 \%)$ & \\
\hline Nivel 4 & $25(19.23 \%)$ & $28(27.18 \%)$ & $53(22.75 \%)$ & \\
\hline
\end{tabular}

Al considerar los centros SINEA, como se observa en la Tabla 8, también encontramos diferencias signficativas entre los dos países. Es el país europeo quien concentra mejores respuestas ante el bullying frente al americano (niveles 3 y 4), tanto para víctimas pasivas como provocativas, aunque en ambos el mayor número de respuestas se concentran en el nivel 2 .

Tabla 8: Posturas morales ante el bullying por países en centros sin estructuras de Equipos de Ayuda.

\begin{tabular}{|c|c|c|c|c|}
\hline \multirow{2}{*}{ Historia } & \multicolumn{3}{|c|}{ SINEA } & \multirow{2}{*}{ Valor-p* } \\
\hline & Brasil & España & Total & \\
\hline \multicolumn{5}{|c|}{ Grupo (HA) } \\
\hline Nivel 1 & $8(6,15 \%)$ & $7(6,8 \%)$ & $34(3,7 \%)$ & \multirow{4}{*}{$<0,01$} \\
\hline Nivel 2 & $71(54,62 \%)$ & $33(32,04 \%)$ & $266(28,91 \%)$ & \\
\hline Nivel 3 & $22(16,92 \%)$ & $33(32,04 \%)$ & $263(28,59 \%)$ & \\
\hline Nivel 4 & $29(22,31 \%)$ & $30(29,13 \%)$ & $357(38,8 \%)$ & \\
\hline \multicolumn{5}{|c|}{ Grupo $(H B)$} \\
\hline Nivel 1 & $12(9,23 \%)$ & $6(5,83 \%)$ & $37(4,01 \%)$ & \multirow{4}{*}{$<0,01$} \\
\hline Nivel 2 & $80(61,54 \%)$ & $49(47,57 \%)$ & $358(38,83 \%)$ & \\
\hline Nivel 3 & $13(10 \%)$ & $20(19,42 \%)$ & $174(18,87 \%)$ & \\
\hline Nivel 4 & $25(19,23 \%)$ & $28(27,18 \%)$ & $353(38,29 \%)$ & \\
\hline
\end{tabular}




\section{DISCUSIÓN}

El trabajo en convivencia ética en la escuela parece que ofrece al alumnado oportunidades de reflexión que les ayuda a dar respuestas ante el bullying con más calidad moral que al alumnado que no dispone de esas oportunidades. El mejor posiconamiento moral ante el bullying del alumnado que se mueve en escenarios donde se visualizan valores de ayuda y compañerismo frente a los del abuso y la imposición se constata en centros CONEA frente a otros similares que no disponen de esas estructuras. Esta significación es más ostensible en el país americano que entre el alumnado espańol, previsiblemente por las diferentes trayectorias de trabajo en convivencia ética que han desarrollado ambos países. Otras investigaciones ya constatan estas diferencias en lo que se refiere al desarrollo del trabajo en valores sociomorales entre el alumnado en ambos países (Avilés, Menin y Tavares, 2019). Recorridos tan diversos justificarían estas diferencias también entre el alumnado de centros CONEA. El trabajo realizado en Brasil en este sentido es muy incipiente y no generalizado. Cualquier intervención sistemática en los centros CONEA marca diferencias evidentes con otros donde no se hace. Por el contrario, en Espańa el trabajo en convivencia viene desarrollándose durante más tiempo. Cualquier centro educativo hoy tiene desarrollado su plan de convivencia.

Sobre la tipología de víctimas, los datos de la investigación apuntan a que un trabajo en convivencia ética en centros CONEA alcanza mejores niveles de desarrollo moral del alumnado ante el bullying, sea con víctimas pasivas -Nivel 3 (30.37\%), Nivel 4 (45.12\%)como ante víctimas provocativas -Nivel 3 (16.63\%), Nivel 4 (43.63\%)-. Esto no resulta contradictorio con los datos obtenidos en Espańa al enfrentar centros con y sin Equipos de Ayuda. El trabajo en convivencia desarrollado a lo largo de estas décadas en los centros espańoles, permite considerar que el contenido en valores está más presente de forma intencional y sistemática en el curriculo y en las prácticas escolares con los estudiantes que lo que está en las escuelas brasileñas, sin un trabajo deliberado en este sentido. De ahí las diferencias en Brasil y la falta de diferencias en Espańa entre centros con y sin Equipos de Ayuda ( $\mathrm{p}=.0 .31$ para HA y $\mathrm{p}=0.21$ para HB).

Si lo que tomamos como punto de comparación son los países, los niveles de desarrollo moral del alumnado que participa en el estudio aporta diferencias signficativas $(\mathrm{p}<0.01$ en victimización pasiva y $\mathrm{p}<0.04$ en victimización provocativa) situando al alumnado español en niveles superiores al brasileńo. Las respuestas que son propiamente morales por principio (Nivel 4) y las que se consideran moralmente conectadas, aunque sea por convención social (Nivel 3), se dan más entre el alumnado de centros espańoles. Si consideramos los centros españoles que tienen estructuras de Equipos de Ayuda, las cifras de conexión moral por convención social (3) duplican a las de los brasileños, (32.04\%) por (16.92\%). Y en un escalón más elevado (Nivel 4) también es superior, al considerar situaciones de bullying con víctima pasiva. En el caso de la víctima provocativa las cifras indican lo mismo, se da una 
mejor y superior interpretación de lo que sucede en los centros CONEA españoles que en los brasileños. En este sentido, se puede encontrar alguna explicación para entender estas diferencias entre países. En Espańa el modelo de Equipos de Ayuda se viene implantando en los centros educativos desde 2006 con niveles de sensibilización y formación tanto del profesorado como del alumnado notables. El recorrido de la filosofía de convivencia y su calado en los centros participantes es mucho mayor que en los de la muestra brasileña, donde apenas se ha logrado implantar el sistema de Equipos de Ayuda en los cuatro últimos ańos. Esto puede explicar una parte importante de las diferencias encontradas.

Desde otra perspectiva, que el alumnado entienda adecudamente las dinámicas de victimización pasiva y provocativa no es fácil y requiere tiempo (Carrascosa, Buelga, Cava y Ortega, 2016). En este sentido, los Equipos de Ayuda pueden ser un vehículo que colabora en ello. Desmontar las formas habituales de desconexión moral del alumnado ante estas situaciones (justificación o culpabilización de las víctimas) para construir otras más conectadas moralmente, exige reflexión e intervención activa por parte de las personas adultas que trabajan con ellos en el día a día y de los propios colegas con posicones más evolucionadas. Entender los mecanismos desencadenantes del acoso por reacciones inadecuadas, por lectura equivocada de situaciones sociales o por trastornos de ansiedad o por TDHA en algunos perfiles de víctimas provocativas, no es un ejercicio sencillo. supone considerar a la víctima provocativa como un actor con necesidades y déficits que no deben mermar su derecho universal a no ser maltratado. Además, eso requiere en el alumnado una verificación que han de comprobar experiencialmente con este tipo de víctimas, acompañado por un trabajo explícito en convivencia ética en la propia escuela, realizado por las personas adultas que acompañan al alumnado, algo que sucede con más frecuencia en contextos en que hay oportunidades para esa reflexión y donde funcionan estructuras como los Equipos de Ayuda, que en otros donde no existe ese trabajo de intermediación cognitiva.

Por otra parte, en la tipología de la victimización el alumnado con frecuencia tiene más dificultades para encajar la pasividad en la autodefensa de quien sufre bullying que los escenarios en los que la víctima participa desencadenando las agresiones, porque en parte esas mismas provocaciones son parte del relato de agresión que llegan a construir (Puértolas y Montiel, 2017). Sin embargo, a las víctimas pasivas los propios colegas les suelen asignar valoraciones de cobardía e indefensión, relegándolas a posiciones de estatus social muy negativas, de no consideración y de rechazo grupal, lo que las excluye de un relato comprensible de agresión. De ahí, que una forma habitual de desconexión moral que suelen utilizar quienes maltratan a una víctima pasiva es la deshumanización (desposeerlas de su condición humana para hacer más soportable lo que les sucede) para hacerlo más comprensible en su relato agresivo (Avilés, Tognetta y Petta, 2020; Tognetta, Avilés y Rosario, 2016). Por eso, es necesario un trabajo en el entorno de esta víctima. Construir a su alrededor un escudo defensivo y un discurso grupal que le devuelva una 
dignidad humanizante y que reivindique su reacción no agresiva, como otra opción también entendible por el grupo; y por otra parte, también con la propia víctima se hace necesario un ejercicio de estimulación y guía de aprendizaje para su autodefensa asertiva.

Por el contrario, la posición de las víctimas provocativas es diferente. Se produce una lectura de rechazo y molestia que podríamos denominar 'activos', lo que termina de explicar en términos de desconexión moral las formas de autoexoneración, la cupabilización (se merece lo que le pasa) o la justificación moral (le pasa eso para corregir su conducta, socialmente no es tan malo que le pase). En este sentido, es especialmente clave hacer entender al grupo las dinámicas reactivas de este perfil de víctima, al alumnado en general y a ellas mismas en particular para asegurar su prevención. Su patrón conductal de provocación-victimizaciónagresión es un proceso que tiene que ser interrumpido para que no les haga daño ni a ellos ni al grupo con el que conviven (Newton, Taylor y Wilson, 2007). En este sentido, la participación de los componentes de los Equipos de Ayuda también se muestra importante (Avilés, 2017; Avilés y Petta, 2018).

Desde otro punto de vista, al considerar el otro perfil de centros, los que no disponen de estructuras de Equipos de Ayuda, aunque es el país europeo el que obtiene mejores respuestas ante el bullying tanto para las víctimas pasivas como provocativas, en ambos países el mayor volumen de elecciones se concentra en el nivel 2 (desconexión moral, aunque sin negación del valor moral en juego). Esto pone de relieve que cuando no se hace presente de forma explícita el discurso ético en los ambientes de convivencia, el alumnado tiende a desconectarse moralmente con más facilidad ante situaciones de victimización, sensiblemente más ante las víctimas provocativas. El alumnado, sin una intermediación específica, usa cualquiera de las formas de desconexión moral (Bandura, 2002). De ahí la importancia de hacer posibles esas formas de explicitar el discurso ético, si puede ser mejor a través del propio alumnado como protavoz, como manera de ofrecer otras explicaciones a por qué una víctima pasiva no es justo que se encuentre en esa posición o que las acciones de una víctima provocativa tal vez no la sitúen como culpable de lo que le sucede. Esto requiere sensibilidad moral y reflexión guiada por parte de personas adultas y de iguales ante el bullying si quieremos reconectar moralmente al alumnado. Aquí la presencia de estructuras como los Equipos de Ayuda puede ser importante. Tener a iguales en los grupos que pongan al alumnado en perspectiva de reflexionar sobre las actuaciones de las víctimas y les ayuden a generar conciencia encontrando vías para minimizar el impacto del bullying en quienes lo sufren, es un elemento crucial que se desprende del estudio para progresar en el desarrollo moral de los iguales (Avilés, Cowie y Alonso, 2019). Para los centros educativos esto será un reto y requerirá, por una parte, posicionamientos de política educativa claros por parte de la insitución y la comunidad educativa, que entiendan que el alumnado debe ser protagonista y coresponsable en la gestión de la convivencia y el acoso, y por otra, actuaciones organizativas, de gestión y de formación para hacerlo posible. 
Por otra parte, se reconocen limitaciones en el estudio referidas a la selección de las muestras de los centros en ambos países, circunscritos a áreas diversas o las diferentes trayectorias culturales y de los sistemas educativos que se comparan y a los que pertenecen los estudiantes, aunque el modelo de apoyo entre iguales, el procedimiento de actuación y la formación que reciben a través de él sean los mismos.

No obstante, las prospectivas apuntan a que las diferencias constatadas entre países y entre centros educativos con y sin estructuras de apoyo entre iguales merecen justificar nuevas investigaciones que abran vías de exploración para analizar el papel sumativo que pueda jugar la intervención sistemática en educación moral del profesorado, inserta en el curriculo de los estudiantes, o el trabajo coordinado que pueden hacer las familias al respecto en la mejora de las elecciones morales de las y los adolescentes frente al acoso.

\section{CONCLUSIÓN}

Los resultados del estudio apuntan datos interesantes respecto del papel de la convivencia ética en la escuela y sobre vías para potenciar en el alumnado mejores niveles de desarrollo moral para lograr minimizar los efectos del acoso entre iguales. Parece que disponer de un discurso constante de convivencia en positivo en el seno de los grupos de iguales en forma de estructura en red marca diferencias respecto a contextos donde ese trabajo y esas estructuras no se dan. El ejemplo de Brasil es significativo. Será necesario seguir extendiendo y generalizando ese modelo que traslada al seno de los grupos una filosofía de convivencia respetuosa y ética para que ayude a quienes puedan ser acosados a mejorar sus posiciones dentro del grupo y mejore el clima y los niveles de conexión moral entre quienes convivan en él. Por otra parte, parece que el discurso que pregonan esas estructuras de apoyo entre iguales es lo significativo además de las propias estructuras. Vease el ejemplo de España. En este sentido, se hace necesario mantener en los centros educativos un trabajo explicito en convivenca ética y el poder que el protagonismo del alumnado puede proyectar como vehículo de trasmisión de valores en los grupos de iguales.

Desde otro punto de vista, el estudio nos aporta otra visión enriquecedora y constructiva. Se aprecian diferencias entre el alumnado sobre cómo se explica las dinámicas de victimización según se enfrenta a un perfil pasivo o provocador de la víctima. Y la existencia de estructuras de apoyo y su discurso mejoran esa explicación, en especial en perfiles más complejos. En este sentido, parece necesario impulsar, contando con el alumnado de los Equipos de Ayuda que puede ser de gran valor, iniciativas para hacer comprender al alumnado las dinámicas bullying y en especial las formas de afrontamiento que las víctimas ponen en marcha en ellas. Una tarea reflexiva y comprometida con los testigos del bullying es imprescindible ante esos comportamientos y reacciones de las víctimas. Por otra parte, se hace necesario un trabajo de empatía y pensamiento en perspectiva con ellos, que les haga entender mejor que la 
renuncia a la violencia como reacción no es sinónimo de cobardía ni de falta de resistencia. $\mathrm{Al}$ contrario, es necesario movilizarlos para que se posicionen con otros valores en defensa de las víctimas, sean pasivas o provocativas.

En definitiva, se pone de manifiesto la importancia del trabajo en convivenca en la escuela como prevención intencional del maltrato entre iguales, y que el alumnado disponga de estructuras de apoyo entre iguales, como los Equipos de Ayuda u otros, que canalicen el potencial de fuerza proactiva en la gestión de su propia convivencia y en la prevención del bullying.

\section{Fuentes de financiación:}

El presente estudio ha sido autofinanciado con recursos propios. Corresponden al autor principal el diseño, interpretación, coordinación y redacción del informe, aunque la responsabilidad es compartida en el manejo contextual y geográfico de los datos.

\section{Aspectos éticos / legales:}

Los autores no han incurrido en aspectos antiéticos, respetando los códigos de investigación con personas, cerciorando la protección de la confidencialidad de la información personal e institucional y asegurando el anonimato de las personas e instituciones involucradas en la muestra.

\section{Conflicto de interés:}

Tampoco se ha producido conflicto de intereses por parte de los autores.

\section{REFERENCIAS}

Avilés, J. Ma (2017). Los Sistemas de Apoyo entre Iguales (SAIs) y su contribución a la convivencia escolar. Innovación Educativa, 27, 5-18. doi: http://dx.doi. org/10.15304/ie.27.4278

Avilés, J. Ma (2019a). Los Sistemas de Apoyo entre Iguales en la escuela. De los Equipos de Ayuda a la Cibermentoría. Barcelona: Graó

Avilés, J. Ma (2019b). Convivir sin bullying. Construyendo relaciones de respeto. Madrid: Narcea

Avilés, J. Ma y Petta, R. (2018). Los Sistemas de Apoyo entre Iguales (SAI) para el fomento de la convivencia en positivo, la mejora del clima de aula y la prevención de situaciones de bullying. La experiencia de Brasil y de Espańa. European Journal 
AVILÉS \& TOGNETTA. Conexiones y desconexiones morales frente al bullying entre alumnado...

of Child Development, Education and Psychopathology, 6(1), 5-17 doi: https://doi. org/10.30552/ejpad.v6i1.58

Avilés, J. Ma y Alonso, Ma N. (2017). Caderno de formaçao das equipes de ajuda. Alunos e alunas: Ensino Fundamental II. Americana, SP. Adonis

Avilés, J. Ma , Torres, N. y Vian, M.V. (2008). Equipos de ayuda, maltrato entre iguales y convivencia escolar. Electronic Journal of Research in Educational Psychology, 6(3), 357-376.

Avilés, J. Ma , Menin, M. S. y Tavares, M. (2019). Evaluación de valores sociomorales en escolares. Investigación comparativa entre Brasil y España. Revista de Educación, 384, 199-228. http://dx.doi.org/10.4438/1988-592X-RE-2019-384-410

Avilés, J. Ma, Cowie, H. y Alonso, N. (2019). El valor de la ayuda. Equipos de Ayuda frente al bullying. Barcelona: Horsori

Avilés, J.Ma , Tognetta, L. y Petta, R. (2020). Actuaciones del profesorado ante el bullying en contextos con y sin Equipos de Ayuda. Estudio en España y Brasil. Revista de Investigación en Psicología, 23(1), 23-41. doi: http://dx.doi.org/10.15381/rinvp.v23i1.18091

Bandura, A. (2002). Selective moral disengagement in the exercise of moral agency. Journal of Moral Education, 31(2), 101-119. http://dx.doi.org/10.1080/0305724022014322

Bandura, A. (2016). Moral disengagement: How people do harm and live with themselves. (1era ed.). New York: Worth.

Carrascosa, L., Buelga, S., Cava, M. J. y Ortega, J. (2016). Violencia escolar entre iguales y ajuste psicosocial: diferencias en función de la frecuencia de la agresión y victimización. En Castejón, J. L. (Coord.). Psicología y Educación: Presente y Futuro, pp. 1463-1471. Recuperado de http://hdl.handle.net/10045/63984

Del Barrio, C., Gutiérrez, H, Barrios, A., van der Meulen, K. y Granizo, L. (2005) Maltrato por abuso de poder entre escolares, ¿de que estamos hablando? Revista Pediatría de Atención Primaria, (VII), 25, 75-100

Díaz-Aguado, M. J. (2006). Sexismo, violencia de género y acoso escolar. Propuestas para una prevención integral de la violencia. Revista de Estudios de Juventud, 73, 38-57.

Gini, G. (2006) Social cognition and moral cognition in bullying: What's wrong? Aggressive behavior, 32, 528-539. http://dx.doi.org/10.1002/ab.20153

Gini, G. Pozzoli,T. y Hauser, M. (2011) Bullies have enhanced moral competence to judge relative to victims, but lack moral compassion. Personality and Individual Differences. 50(5), 603-608. doi: http://dx.doi.org/10.1016/j.paid.2010.12.002

Gini, G., Thornberg, R. y Pozzoli, T. (2020). Individual moral disengagement and bystander behavior in bullying: The role of moral distress and collective moral disengagement. Psychology of Violence, 10(1), 38-47. http://dx.doi.org/10.1037/vio0000223 
Juvonen, J. y Galvan, A. (2008) Peer contagion in involuntary social groups: Lessons from research on bullying. En Pristein, M. y Dodge, K. (Eds.) Peer influence processes among youth. New York: Guilford Press.

Kohlberg, L. (1992). Psicologia del desarrollo moral. Bilbao: Desclée de Brouwer.

Newton, C., Taylor, G. y Wilson, D. (2007). Creating Circles of Friends, Inclusive Solutions. Recuperado de [https://inclusive-solutions.com/circles/circle-of-friends/childrenhelping-children/.

Piaget (1932/1994). O juizo moral na criança. São Paulo, Summus.

Puértolas, A. y Montiel, I. (2017). Bullying en la educación secundaria: una revisión sobre las características de las víctimas y las víctimas-acosadores y las consecuencias de su victimización. Revista de Victimología, 5, 85-128. Recuperado de http://www. huygens.es/journals/index.php/revista-de-victimologia/article/view/91

Salmivalli, C. y Voeten, M. (2004). Connections between atitudes, group norms, and behaviors associated with bullying in schools. International Journal oh Behavioral Development, 28, 246-258. https://doi.org/10.1080\%2F01650250344000488

Salmivalli, C. y Peets, K. (2009). Bullies, victims, and bully-victim relationship. En K. Rubin W. Bukowski y B. Laursen (Eds.), Social, emotional, and personality development in context. Handbook of peer interactions, relationships, and groups (p. 322-340). The Guilford Press. https://psycnet.apa.org/record/2008-19117-018

Sutton, J., Smith, P.K. y Swettenham, J. (1999). Bullying and "theory of mind": a critique of the "social skills deficit" view of anti-social behaviour. Social developmental 8, 117 127. doi: http://dx.doi.org/10.1111/1467-9507.00083

Tognetta, L. y Rosário, P. (2013). Bullying: dimensóes psicológicas no desenvolvimento moral. Estudos em Avaliação Educacional, 24 (56), 106-137. doi: http://dx.doi. org/10.18222/eae245620132736

Tognetta, L., Avilés, J.M. y Rosario, P. (2016). Bullying, un problema moral: representaciones de sí mismo y desconexiones morales. Revista de Educación, 373, 9-34. doi: http:// dx.doi.org/10.4438/1988-592X-RE-2016-373-319

Tognetta, L.R.P., Avilés, J.M., Rosário, P. y Alonso, N. (2015). Desengajamentos morais, autoeficácia e bullying: a trama da convivência. Estudios e investigación em psicologia y educación. 2 (1), 30-34. http://dx.doi.org/10.17979/reipe.2015.2.1.714.

Recibido: 09 de enero de 2021 Aceptado: 18 de febrero de 2021 